\title{
Risk factors analysis of acute kidney injury following open thoracic aortic surgery in the patients with or without acute aortic syndrome: a retrospective study
}

Xiaochun $\mathrm{Ma}^{1,2^{*+}} \mathbb{D}$, Jinzhang $\mathrm{Li}^{3 \dagger}$, Yan Yun ${ }^{4+}$, Diming Zhao ${ }^{1,2}$, Shanghao Chen ${ }^{1,2}$, Huibo $\mathrm{Ma}^{5}$, Zhengjun Wang ${ }^{1,2}$, Haizhou Zhang ${ }^{1,2}$, Chengwei Zou ${ }^{1,2}$ and Yuqi Cui ${ }^{6,7^{*}}$

\begin{abstract}
Background: The acute kidney injury (AKI) remains a frequent complication following open thoracic aortic surgery (OTAS) and worsens the postoperative prognosis. It remains unclear that whether the predictors of AKI following OTAS are different in the patients with or without acute aortic syndrome (AAS).

Methods: Preoperative and intraoperative variables were compared between the patients with or without AKI, and were further analyzed for identifying the potential predictors of postoperative AKI. Subgroup analysis was conducted in the patients with or without AAS, respectively.

Results: AKI after OTAS occurred in $57.6 \%$ of the overall cohort, $70.1 \%$ of the patients with AAS and $46.7 \%$ of the patients without AAS. In the multivariate analysis, history of hypertension (OR 1.011,95\% Cl: [1.001-1.022], $p=0.04$ ), preoperative platelet (OR 0.995, 95\% Cl: [0.991-0.999], $p=0.006$ ) and operation time (OR 1.572, 95\% Cl: [1.355-1.823], $p<0.001)$ were identified as independent predictors of postoperative AKI for the overall cohort; CPB time (OR 1.020, 95\% Cl: [1.009-1.031], $p<0.001$ ) and preoperative LMR (OR 0.823, 95\% Cl: [0.701-0.966], $p=0.02$ ) as independent predictors for the patients with AAS; age (OR 1.045, 95\% Cl: [1.015-1.076], $p=0.003)$, preoperative platelet (OR 0.993, 95\% Cl: [0.988-0.998], $p=0.04$ ) and operation time (OR 1.496, 95\% Cl: [1.166-1.918], $p=0.002$ ) as independent predictors for the patients without AAS.

Conclusions: The patients with AAS carry a higher risk for postoperative AKI compared with those without AAS. The predictive factors for postoperative AKI after OTAS are different for AAS- and non-AAS subgroups and operation time, CPB time and preoperative platelet are modifiable predictors of AKI.
\end{abstract}

Keywords: Acute aortic syndrome, Acute kidney injury, Independent predictor, Open thoracic aortic surgery

\footnotetext{
*Correspondence: mxcmxc2008@163.com; cuiyu@health.missouri.edu

${ }^{+}$Xiaochun Ma, Jinzhang Li and Yan Yun contributed equally to this work.

1 Department of Cardiovascular Surgery, Shandong Provincial Hospital

affiliated to Shandong First Medical University, No.324 Jingwu Road, Jinan

250021, Shandong, China

${ }^{6}$ Department of Cardiology, Shandong Provincial Hospital affiliated to

Shandong First Medical University, No.324 Jingwu Road, Jinan 250021,

Shandong, China

Full list of author information is available at the end of the article
}

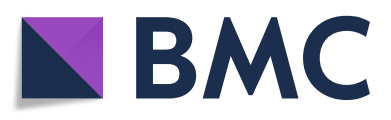

(- The Author(s). 2020 Open Access This article is licensed under a Creative Commons Attribution 4.0 International License, which permits use, sharing, adaptation, distribution and reproduction in any medium or format, as long as you give appropriate credit to the original author(s) and the source, provide a link to the Creative Commons licence, and indicate if changes were made. The images or other third party material in this article are included in the article's Creative Commons licence, unless indicated otherwise in a credit line to the material. If material is not included in the article's Creative Commons licence and your intended use is not permitted by statutory regulation or exceeds the permitted use, you will need to obtain permission directly from the copyright holder. To view a copy of this licence, visit http://creativecommons.org/licenses/by/4.0/ The Creative Commons Public Domain Dedication waiver (http://creativecommons.org/publicdomain/zero/1.0/) applies to the data made available in this article, unless otherwise stated in a credit line to the data. 


\section{Introduction}

The AKI complicates $10-70 \%$ patients undergoing OTAS, depending on the distinct definitions of AKI [1-7]. This complication predicts a dismal prognosis and increases the postoperative mortality and morbidity. Despite the recent advancements in cardiopulmonary bypass $(\mathrm{CPB})$, anesthesia and intensive care, the incidence of AKI following OTAS remains high [8]. The AKI contemporarily represents a huge challenge for cardiovascular surgeons as no definite therapy has thus been developed. Though renal replacement therapy (RRT) is a feasible therapeutic option, modifying those potential risk factors could fundamentally prevent the occurrence of AKI and improves the short- and longterm outcomes [2, 9-12].

The primary objective of this retrospective cohort study was to clarify that whether different risk factors predict AKI after OTAS in the patients with or without AAS.

\section{Materials and methods Study design and participants}

This single-center retrospective cohort study was approved by the Ethics Committee of Shandong Provincial Hospital and the written informed consent was waived due to the retrospective design. 399 consecutive patients were eventually recruited who underwent the elective or emergent OTAS between January 1, 2015 to December 31,2018 . The patients were excluded from the cohort who died within $72 \mathrm{~h}$ after operations or whose preoperative and intraoperative data were insufficient or who had preoperative renal insufficiency or previous renal transplantation (Fig. 1). The study was conducted following the Good Clinical Practice (GCP) as well as principles of the Declaration of Helsinki. The clinical data of those patients were carefully collected from admission to discharge. All the operations were completed by an identical surgical team. In this study, AAS represents a series of acute and catastrophic aortic lesions including acute aortic dissection, intramural hematoma, penetrating aortic ulcer, and rupture of aorta due to aortic aneurysm or trauma. Non-AAS represents the non-acute and non-catastrophic thoracic aortic lesions that require elective surgical treatment. The included 399 patients were divided into the AAS subgroup and non-AAS subgroup for further analysis. Potential demographic or perioperative variables considered associated with postoperative AKI were included on the basis of clinical experiences and literature review.

\section{Definition of AKI following OTAS and postoperative outcomes}

The primary outcome of this cohort study was AKI after OTAS. The diagnosis of AKI was established according to the modified Kidney Disease: Improving Global Outcomes guidelines (KDIGO) [13]. The postoperative AKI was defined as an increase of $>50 \%$ of the baseline within the first 7 days or $>0.3 \mathrm{mg} / \mathrm{dL} \quad(26.5 \mathrm{umol} / \mathrm{L})$ within the first $48 \mathrm{~h}$ in the postoperative serum creatinine (Scr). The baseline Scr was ascertained based on the latest preoperative tests. And the AKI was further graded as stage I, II and III according to the KDIGO classification and the patients were categorized in line with their highest levels of postoperative Scr. Besides, the postoperative outcomes attributable to AKI included RRT, length of ICU stay and ICU stay $>7$ days, intubation time and time $>5$ days, stroke, redo surgery and in-hospital mortality.

\section{Surgical details}

The Surgical details were summarized in the supplementary material.

\section{Statistical analysis}

Statistical analysis was performed using SPSS Statistics 25.0. Categorical variables were expressed as frequencies (n) with percentages (\%). Continuous variables were expressed as mean \pm standard deviation (SD) or median (quartile), depending on the data dispersion. The t-test was used if a continuous variable conformed normal distribution. And non-parametric Mann-Whitney $U$ test was applied if a continuous variable was consistent with skewed distribution. A chi-squared or a Fisher's exact test was used for categorical variables when necessary. The univariate and multivariate analyses were performed using binary logistic regression analysis. The candidate univariates (with a p valve less than 0.1 ) as well as those possible predictive variables were included in the multivariate predictive model. And the predictive model was further evaluated using the receiver operating curve (ROC) and the Hosmer-Lemeshow test. Except that the overall cohort were analyzed as above-mentioned, the subgroup analyses were additionally conducted for the patients with or without AAS. All the statistical tests were two-sided and a p valve less than 0.05 was considered statistically significant.

\section{Results \\ Patient characteristics}

A total of 399 patients were enrolled in this retrospective cohort study and the patient characteristics of overall cohort, AAS subgroup and non-AAS subgroup were shown respectively in the supplementary material. The patients were excluded who died within $72 \mathrm{~h}$ after operations $(n=4)$, whose preoperative and intraoperative data were insufficient $(n=3)$ as well as who had preoperative renal insufficiency and previous renal transplantation $(n=8)$. The causes of death of these 4 patients included 


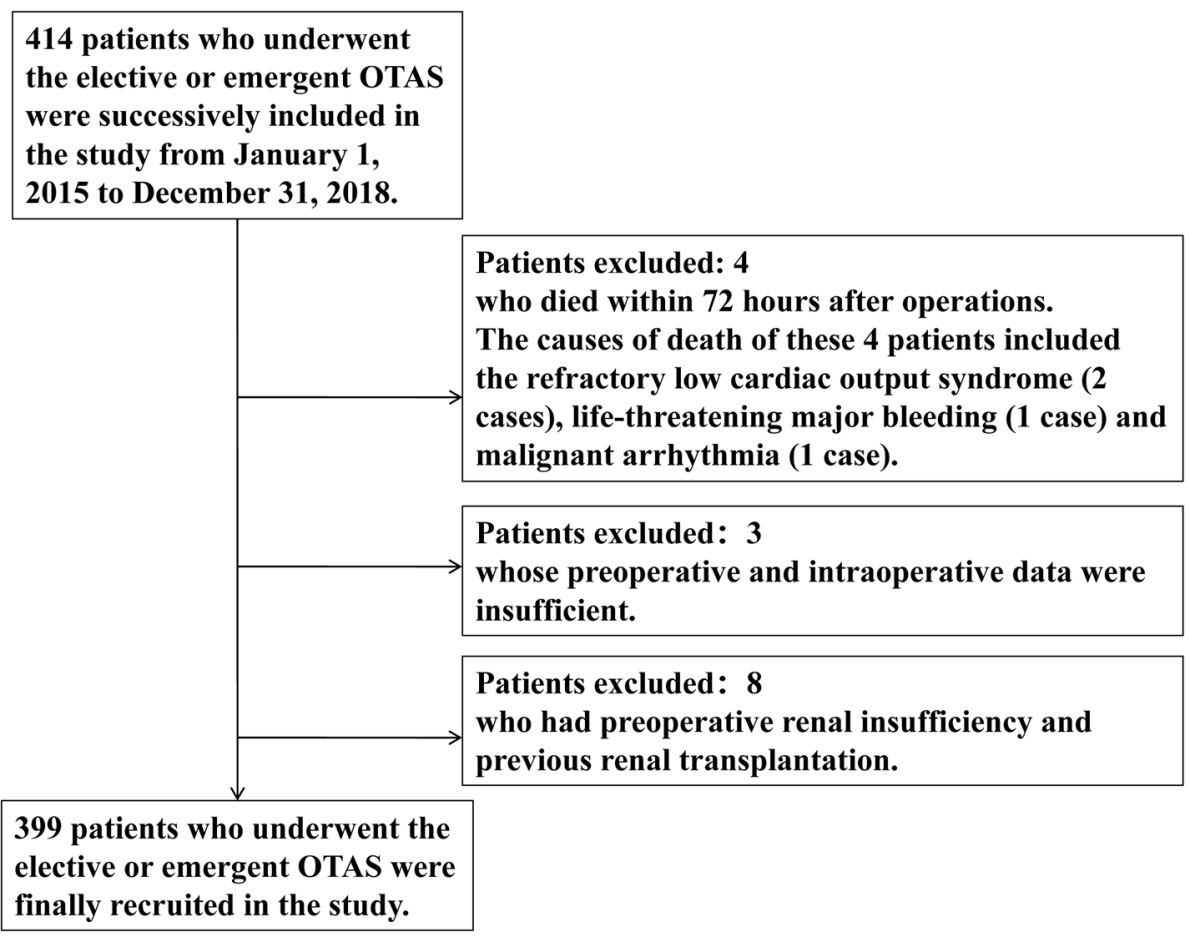

Fig. 1 Flow chart of participants inclusion and exclusion. Figure 1 depicts the process of participants inclusion and exclusion

the refractory low cardiac output syndrome $(n=2)$, lifethreatening major bleeding $(n=1)$ and malignant arrhythmia $(n=1)$. The surgical options of overall cohort were presented in the supplementary Table 1. And the surgical options of AAS subgroup and nonAAS subgroup were showed in supplementary Table 2 and supplementary Table 3, respectively.

Incidence of AKI after open thoracic aortic surgery Postoperative AKI in the overall cohort

According to the modified KDIGO's Criteria, the incidence of AKI after aortic operation was 57.6\% (230 in $399)$, among whom 153 patients (38.3\%) met the criteria of stage 1, 49 patients (12.3\%) satisfied the criteria of stage 2 , and 28 patients $(7.0 \%)$ reached the stage 3 (Table 1).

\section{Postoperative AKI in the patients with AAS}

In the subgroup of patients with AAS, the incidence of postoperative AKI was evidently higher than that of the overall cohort $(p<0.01) .131$ in $187(70.1 \%)$ developed the postoperative AKI, among whom 71 patients (38.0\%) met the criteria of stage 1, 36 patients (19.3\%) satisfied the criteria for stage $2 \mathrm{AKI}$, and 24 patients (12.8\%) met reached the stage 3 (Table 3 ).
Postoperative AKI in the patients without AAS

Compared with the AAS subgroup, the incidence of postoperative AKI was markedly reduced in the non-AAS subgroup $(p<0.001)$. Of 212 patients in the non-AAS subgroup, 99 (46.7\%) patients developed the postoperative AKI. 82 patients (38.7\%) met the criteria of stage 1,13 patients $(6.1 \%)$ reached the criteria for stage 2, 4 patients $(1.9 \%)$ were in stage 3 (Table 5).

\section{Risk factors of postoperative AKI after open thoracic aortic surgery \\ Risk factors of postoperative AKI for the overall cohort}

Demographic and perioperative data of patients grouped by the severity of the KDIGO classification were present in Table 1. There were significant statistical differences in a series of variables including age, weight, BMI, history of hypertension, preoperative platelet, lymphocyte, monocyte, neutrophil and several other laboratory variables, Marfan syndrome, aortic dissection (as well as the Stanford type-A aortic dissection), intraoperative infusion of erythrocytes, fresh frozen plasma and platelets, combined valvular surgery and all the surgical data (Table 1).

The incidences of CRRT, ICU stay $>7$ days, intubation time $>5$ days, stroke, redo surgery and in-hospital mortality were all significantly higher among the patients with AKI than those without AKI. The time of ICU stay 
Table 1 Characteristics of the Overall Cohort

\begin{tabular}{|c|c|c|c|c|c|c|}
\hline & No AKI & AKI & & & P1 Value* & P2 Value ${ }^{\#}$ \\
\hline & & Stage 1 & Stage 2 & Stage 3 & & \\
\hline Patient population (n) & 169 & 153 & 49 & 28 & & \\
\hline Demographic data & & & & & & \\
\hline Age (y) & $52.0(18.0)$ & $54.0(16.5)$ & $50.0(13.0)$ & $41.5(17.5)$ & 0.9 & 0.004 \\
\hline Sex, male (n) & $112(66.3 \%)$ & $116(75.8 \%)$ & $34(69.4 \%)$ & $21(75.0 \%)$ & 0.08 & 0.3 \\
\hline Height (cm) & $170.0(13.0)$ & $170.0(8.5)$ & $170.0(14.0)$ & $169.0(10.0)$ & 0.7 & 1.0 \\
\hline Weight (kg) & $70.0(16.0)$ & $72.0(18.0)$ & $74.0(24.0)$ & $75.0(20.0)$ & 0.002 & 0.02 \\
\hline BMI $\left(\mathrm{kg} / \mathrm{m}^{2}\right)$ & $24.6(5.1)$ & $25.7(5.8)$ & $25.2(4.9)$ & $27.4(5.8)$ & 0.003 & 0.009 \\
\hline Medical history & & & & & & \\
\hline Diabetes (n) & $4(2.4 \%)$ & $4(2.6 \%)$ & $3(6.1 \%)$ & $1(3.6 \%)$ & 0.5 & 0.6 \\
\hline Hypertension (n) & $74(43.8 \%)$ & $85(55.6 \%)$ & $34(69.4 \%)$ & $23(82.1 \%)$ & $<0.001$ & $<0.001$ \\
\hline Chronic obstructive pulmonary disease (n) & $4(2.4 \%)$ & $6(3.9 \%)$ & $1(2.0 \%)$ & $0(0.0 \%)$ & 0.7 & 0.6 \\
\hline Previous myocardial infarction (n) & $1(0.6 \%)$ & $0(0.0 \%)$ & $0(0.0 \%)$ & $0(0.0 \%)$ & 0.2 & 0.7 \\
\hline Peripheral vascular disease (n) & $13(7.7 \%)$ & $11(7.2 \%)$ & $5(10.2 \%)$ & $1(3.6 \%)$ & 0.9 & 0.8 \\
\hline Smoking (n) & $57(33.7 \%)$ & $59(38.6 \%)$ & 19 (38.8\%) & $12(42.9 \%)$ & 0.3 & 0.7 \\
\hline Preoperative laboratory tests & & & & & & \\
\hline Platelet (109/L) & $197.0(71.0)$ & $186.5(71.8)$ & $199.0(65.5)$ & $166.5(68.3)$ & 0.006 & 0.01 \\
\hline Lymphocyte (109/L) & $1.63(0.74)$ & $1.58(0.90)$ & $1.18(0.72)$ & $1.48(0.90)$ & 0.004 & 0.001 \\
\hline Monocyte (109/L) & $0.44(0.30)$ & $0.51(0.40)$ & $0.79(0.78)$ & $0.91(0.70)$ & $<0.001$ & $<0.001$ \\
\hline Neutrophil (109/L) & $4.22(2.95)$ & $4.86(4.30)$ & $7.51(5.64)$ & $10.1(5.3)$ & $<0.001$ & $<0.001$ \\
\hline Hyperlipidemia (n) & $55(34.4 \%)$ & $44(31.0 \%)$ & $7(14.9 \%)$ & $10(43.5 \%)$ & 0.2 & 0.04 \\
\hline Retinol binding protein (mg/L) & $35.7(16.2)$ & $37.8(18.7)$ & $26.4(24.7)$ & $37.3(22.5)$ & 0.7 & 0.007 \\
\hline Urinary protein & & & & & & \\
\hline$+/-$ & $17(11.6 \%)$ & $25(18.8 \%)$ & $10(23.8 \%)$ & $4(20.0 \%)$ & 0.05 & 0.2 \\
\hline+ & $14(9.5 \%)$ & $20(15.0 \%)$ & $11(26.2 \%)$ & $8(40.0 \%)$ & 0.01 & 0.005 \\
\hline++ & $1(0.7 \%)$ & $2(1.5 \%)$ & $2(4.8 \%)$ & $1(5.0 \%)$ & 0.2 & 0.3 \\
\hline INR & $1.04(0.12)$ & $1.04(0.1)$ & $1.09(0.15)$ & $1.10(0.2)$ & 0.05 & 0.02 \\
\hline Preoperative renal function & & & & & & \\
\hline Preoperative SCr ( $\mu \mathrm{mol} / \mathrm{L})$ & $70.4(21.79)$ & $73.6(23.1)$ & $66.0(26.3)$ & $76.0(45.7)$ & 0.3 & 0.07 \\
\hline$>106(\mathrm{n})$ & $11(6.5 \%)$ & $13(8.5 \%)$ & $4(8.2 \%)$ & $8(28.6 \%)$ & 0.1 & 0.002 \\
\hline $\mathrm{eGFR}(\mathrm{mL} / \mathrm{min} / 1.73 \mathrm{~m} 2)$ & $99.8(22.0)$ & $97.2(21.2)$ & $103.4(18.2)$ & $94.4(39.5)$ & 0.2 & 0.08 \\
\hline Preoperative cardiovascular status & & & & & & \\
\hline Marfan syndrome (n) & $14(8.3 \%)$ & $3(2.0 \%)$ & $1(2.0 \%)$ & $1(3.6 \%)$ & 0.005 & 0.04 \\
\hline Aortic dissection (n) & $56(33.1 \%)$ & $71(46.4 \%)$ & $36(73.5 \%)$ & $24(85.7 \%)$ & $<0.001$ & $<0.001$ \\
\hline Stanford type-A aortic dissection ( $n$ ) & $53(31.4 \%)$ & $71(46.4 \%)$ & $34(69.4 \%)$ & $24(85.7 \%)$ & $<0.001$ & $<0.001$ \\
\hline Intraoperative blood product use & & & & & & \\
\hline Erythrocytes (u) & $2.0(2.0)$ & $4.0(3.9)$ & $4.0(6.0)$ & $4.0(4.5)$ & 0.001 & $<0.001$ \\
\hline Fresh frozen plasma $(\mathrm{ml})$ & $400.0(200.0)$ & $400.0(150.0)$ & $400.0(375.0)$ & $600.0(500.0)$ & 0.06 & 0.002 \\
\hline Platelets (u) & $1.0(0.0)$ & $1.0(1.0)$ & $1.0(1.0)$ & $2.0(1.0)$ & 0.002 & $<0.001$ \\
\hline Surgical details & & & & & & \\
\hline Involving the aortic arch (n) & $79(46.7 \%)$ & $97(63.4 \%)$ & $38(77.6 \%)$ & $26(92.9 \%)$ & $<0.001$ & $<0.001$ \\
\hline Involving the descending aorta (n) & $43(25.4 \%)$ & $60(39.2 \%)$ & $33(67.3 \%)$ & $24(85.7 \%)$ & $<0.001$ & $<0.001$ \\
\hline CPB duration(min) & $133.0(75.5)$ & $165.0(79.0)$ & $201.0(67.5)$ & $220.0(52.0)$ & $<0.001$ & $<0.001$ \\
\hline DHCA or MHCA (n) & $58(34.3 \%)$ & $76(49.7 \%)$ & $34(69.4 \%)$ & $25(89.3 \%)$ & $<0.001$ & $<0.001$ \\
\hline Aortic cross-clamp time (min) & $86.0(27.0)$ & $90.0(31.0)$ & $96.0(32.0)$ & $113.0(32.0)$ & $<0.001$ & $<0.001$ \\
\hline Operation time (h) & $6.0(2.0)$ & $7.0(2.0)$ & $8.5(2.0)$ & $9.0(2.5)$ & $<0.001$ & $<0.001$ \\
\hline Combined surgery & & & & & & \\
\hline Valvular surgery (n) & $94(55.6 \%)$ & $67(43.8 \%)$ & $10(20.4 \%)$ & $3(10.7 \%)$ & $<0.001$ & $<0.001$ \\
\hline
\end{tabular}


Table 1 Characteristics of the Overall Cohort (Continued)

\begin{tabular}{|c|c|c|c|c|c|c|}
\hline & \multirow[t]{2}{*}{ No AKI } & \multicolumn{3}{|l|}{ AKI } & \multirow[t]{2}{*}{ P1 Value } & \multirow[t]{2}{*}{ P2 Value } \\
\hline & & Stage 1 & Stage 2 & Stage 3 & & \\
\hline \multicolumn{7}{|l|}{ Outcomes } \\
\hline Renal replacement therapy (n) & $0(0.0 \%)$ & $0(0.0 \%)$ & $0(0.0 \%)$ & $12(42.9 \%)$ & 0.003 & $<0.001$ \\
\hline Length of ICU stay (day) & $3.0(2.0)$ & $4.0(2.5)$ & $5.0(4.0)$ & $10.5(11.0)$ & $<0.001$ & $<0.001$ \\
\hline$>7 d(n)$ & $19(11.2 \%)$ & $20(13.1 \%)$ & $17(34.7 \%)$ & $23(82.1 \%)$ & $<0.001$ & $<0.001$ \\
\hline Intubation time (h) & $15.0(9.0)$ & $17.0(27.5)$ & $38.0(72.5)$ & $136.0(110.3)$ & $<0.001$ & $<0.001$ \\
\hline$>5 d(n)$ & $4(2.4 \%)$ & $5(3.3 \%)$ & $6(12.2 \%)$ & $17(60.7 \%)$ & $<0.001$ & $<0.001$ \\
\hline Stroke $(n)$ & $0(0.0 \%)$ & $4(2.6 \%)$ & $0(0.0 \%)$ & $2(7.1 \%)$ & 0.04 & 0.01 \\
\hline Redo surgery (n) & $4(2.4 \%)$ & $6(3.9 \%)$ & $6(12.2 \%)$ & $6(21.4 \%)$ & 0.02 & $<0.001$ \\
\hline In-hospital mortality (n) & $2(1.2 \%)$ & $1(0.7 \%)$ & $1(2.1 \%)$ & $8(28.6 \%)$ & 0.07 & $<0.001$ \\
\hline
\end{tabular}

and intubation also markedly increased in the patients with AKI compared those without AKI (Table 1).

In the univariate analysis of risk factors for the overall cohort, body weight, history of hypertension, Marfan syndrome, preoperative platelet, lymphocyte, monocyte and neutrophil, neutrophil to lymphocyte ratio (NLR), lymphocyte to monocyte ratio (LMR), preoperative urinary protein positive $(+,++)$, Marfan syndrome, aortic dissection (Stanford type-A aortic dissection), intraoperative fresh frozen plasma use, intraoperative involvement of aortic arch and descending aorta, $\mathrm{CPB}$ time, aortic cross-clamp time, operation time, DHCA or MHCA and concurrent valve surgery were candidate variables (Table 2).

\section{Risk factors of postoperative AKI for the patients with AAS}

For the subgroup of patients with AAS, there existed significant statistical differences in those variables including male gender, weight, history of hypertension, preoperative lymphocyte, monocyte and neutrophil and several other laboratory variables, $\mathrm{CPB}$ time, aortic cross-clamp time, operation time and combined CABG (Table 3).

Similarly as above, the proportions of CRRT, ICU stay $>7$ days, intubation time $>5$ days, stroke, redo surgery and in-hospital mortality were all higher in the AKI group. Consistently, the durations of ICU stay and intubation were evidently increased in the patients with AKI in comparison with those without AKI (Table 3).

When it comes to the univariate analysis of risk factors for the AAS cohort, male gender, weight, preoperative lymphocyte, monocyte, neutrophil, NLR, LMR, CPB time, aortic cross-clamp time and operation time were included in the list of plausible variables (Table 4).

\section{Risk factors of postoperative AKI for the patients without AAS}

For the non-AAS subgroup, those variables reached statistical significance that include age, history of hypertension, preoperative platelet and eGFR, previous cardiovascular surgery, Marfan syndrome, intraoperative perfusion of fresh frozen plasma and platelets, operation involving the descending aorta, $\mathrm{CPB}$ time, aortic crossclamp time, DHCA or MHCA and operation time (Table 5).

For the patients in the non-AAS subgroup, the rates of CRRT, ICU stay $>7$ days, intubation time $>5$ days and in-hospital mortality were evidently enhanced when compared to those without AKI. And the ICU length of stay also elevated in the patients with AKI (Table 5).

As for the univariate analysis of risk factors for the non-AAS cohort, several potential risk factors have been identified that consist of age, preoperative platelet and platelet to lymphocyte ratio (PLR), CPB time and operation time (Table 6).

\section{The establishment of predictive model for postoperative AKI}

Predictive model for postoperative AKI in the overall cohort Those independent predictors of postoperative AKI after OTAS included history of hypertension (OR 1.011, 95\% CI: [1.001-1.022], $p=0.04$ ), preoperative platelet (OR 0.995, 95\% CI: [0.991-0.999], $p=0.006$ ) and operation time (OR 1.572, 95\% CI: [1.355-1.823], $p<0.001$ ). The equation obtained by binary Logistic regression analysis was as follows: $Y=0.011 \times$ history of hypertension$0.005 \times$ preoperative platelet $+0.452 \times$ operation time4.814. The predictive model was evaluated by the ROC curve. The area under a curve (AUC) of the ROC curve 
Table 2 Univariate Analysis of Risk Factors for the Overall Cohort

\begin{tabular}{|c|c|c|c|}
\hline Variable & Odds Ratio & 95\% Confidence Interval & $P$ Value \\
\hline Age (y) & 1.01 & $0.99-1.02$ & 0.6 \\
\hline Sex, male & 1.48 & $0.95-2.28$ & 0.08 \\
\hline Weight & 1.02 & $1.01-1.04$ & 0.006 \\
\hline Hypertension & 2.07 & $1.38-3.10$ & $<0.001$ \\
\hline Preoperative platelet & 1.00 & $0.99-1.00$ & 0.006 \\
\hline Preoperative lymphocyte & 0.64 & $0.46-0.88$ & 0.007 \\
\hline Preoperative monocyte & 3.76 & $2.02-7.00$ & $<0.001$ \\
\hline Preoperative neutrophil & 1.16 & $1.08-1.23$ & $<0.001$ \\
\hline NLR & 1.17 & $1.10-1.25$ & $<0.001$ \\
\hline LMR & 0.88 & $0.81-0.96$ & 0.003 \\
\hline Preoperative urinary protein + or ++ & 2.43 & $1.30-4.53$ & 0.005 \\
\hline Marfan syndrome & 0.25 & $0.09-0.70$ & 0.008 \\
\hline Aortic dissection & 2.67 & $1.77-4.04$ & $<0.001$ \\
\hline Standford type-A aortic dissection & 2.80 & $1.84-4.24$ & $<0.001$ \\
\hline Intraoperative fresh frozen plasma use & 1.00 & $1.00-1.00$ & 0.01 \\
\hline Involving the aortic arch & 2.66 & $1.76-4.02$ & $<0.001$ \\
\hline Involving the descending aorta & 3.03 & $1.97-4.67$ & $<0.001$ \\
\hline CPB duration & 1.01 & $1.01-1.02$ & $<0.001$ \\
\hline DHCA or MHCA & 2.72 & $1.80-4.11$ & $<0.001$ \\
\hline Aortic cross-clamp time & 1.02 & $1.01-1.02$ & $<0.001$ \\
\hline Operation time & 1.63 & $1.41-1.89$ & $<0.001$ \\
\hline Combined valvular surgery & 0.43 & $0.28-0.64$ & $<0.001$ \\
\hline
\end{tabular}

Abbreviations: NLR Neutrophil to Lymphocyte Ratio, LMR Lymphocyte to Monocyte Ratio, SCr Serum Creatinine, CPB Cardiopulmonary Bypass, DHCA Deep Hypothermia and Circulatory Arrest, MHCA Median Hypothermia and Circulatory Arrest

was 0.750 ( $p<0.001,95 \%$ CI: $0.702-0.798)$ (Fig. 2). When the maximum value of the Youden index was 0.409 , at this time $\mathrm{Y}=0.031$, the sensitivity of the model was $76.0 \%$ and the specificity was $64.9 \%$.

\section{Predictive model for postoperative $A K I$ in the patients with AAS}

For the patients with AAS, CPB time (OR 1.020, 95\% CI: [1.009-1.031], $p<0.001$ ) and preoperative LMR (OR 0.823, 95\% CI: [0.701-0.966], $p=0.02$ ) independently predicted the incidence of postoperative AKI. The equation obtained by binary Logistic regression analysis was: $\mathrm{Y}=0.020 \times \mathrm{CPB}$ time- $0.195 \times \mathrm{LMR}-5.601$. The AUC of ROC curve was $0.784(p<0.001,95 \%$ CI: 0.714-0.855) (Fig. 3). When the maximum value of the Youden index reached 0.467 , at this time $\mathrm{Y}=1.056$, the sensitivity of the model was $66.7 \%$ and the specificity was $80.0 \%$.

\section{Predictive model for postoperative AKI in the patients without acute aortic syndrome}

For the patients without AAS, age (OR 1.045, 95\% CI: [1.015-1.076], $p=0.003)$, preoperative platelet (OR
0.993, 95\% CI: [0.988-0.998], $p=0.04)$ and operation time (OR 1.496, 95\% CI: [1.166-1.918], $p=0.002$ ) independently predicted the incidence of postoperative AKI. The equation obtained by binary Logistic regression analysis was: $\mathrm{Y}=0.044 \times$ age $-0.006 \times$ preoperative platelet $0.403 \times$ operation time-3.751. The AUC of ROC curve (see Fig. 4) was 0.719 ( $P<0.001$, 95\% CI: $0.651-$ 0.787 ). When the maximum value of the Youden index reached 0.467 , at this time $\mathrm{Y}=1.0564$, the sensitivity of the model was $81.4 \%$ and the specificity was $52.2 \%$.

\section{Discussion}

Prospective or retrospective studies have previously found that the incidence of AKI after thoracic aortic surgery is approximately $18-67 \%$, depending on the definition of AKI [1-7]. Some of these prior papers recruited a highly heterogeneous group of patients, regardless of disease types and surgical options. And the other papers were designed to study on a specific group of patients, such as the patients undergoing elective or emergent OTAS, overweight patients who underwent total aorta replacement (TAR) combined with frozen 
Table 3 Characteristics of the patients with acute aortic syndrome

\begin{tabular}{|c|c|c|c|c|c|c|}
\hline & No AKI & AKI Stage & & & $P 1$ Value & P2 Value \\
\hline & & 1 & 2 & 3 & & \\
\hline Patient population (n) & 56 & 71 & 36 & 24 & & \\
\hline Demographic data & & & & & & \\
\hline Age (y) & $48.0(16.5)$ & $48.0(15.0)$ & $48.0(13.5)$ & $41.0(8.3)$ & 0.2 & 0.07 \\
\hline Sex, male (n) & $31(55.4 \%)$ & $52(73.2 \%)$ & $24(66.7 \%)$ & $18(75.0 \%)$ & 0.03 & 0.1 \\
\hline Height (cm) & $170.0(12.8)$ & $171.0(7.0)$ & $172.0(10.5)$ & $170.0(13.0)$ & 0.4 & 0.5 \\
\hline Weight (kg) & $71.9 \pm 10.2$ & $78.0 \pm 12.6$ & $77.1 \pm 14.4$ & $77.8 \pm 13.4$ & 0.003 & 0.07 \\
\hline $\mathrm{BMI}\left(\mathrm{kg} / \mathrm{m}^{2}\right)$ & $25.5 \pm 2.8$ & $25.7 \pm 5.6$ & $26.4 \pm 3.9$ & $28.2 \pm 3.7$ & 0.3 & 0.2 \\
\hline Medical history & & & & & & \\
\hline Diabetes $(n)$ & $2(3.6 \%)$ & $1(1.4 \%)$ & $2(5.6 \%)$ & $1(4.2 \%)$ & 0.9 & 0.7 \\
\hline Hypertension (n) & $38(67.9 \%)$ & $46(64.8 \%)$ & $30(83.3 \%)$ & $23(95.8 \%)$ & 0.3 & 0.009 \\
\hline Chronic obstructive pulmonary disease ( $\mathrm{n}$ ) & $1(1.8 \%)$ & $1(1.4 \%)$ & $0(0.0 \%)$ & $0(0.0 \%)$ & 0.5 & 0.8 \\
\hline Peripheral vascular disease $(n)$ & $4(7.1 \%)$ & $5(7.0 \%)$ & $4(11.1 \%)$ & $1(4.2 \%)$ & 0.9 & 0.8 \\
\hline Smoking (n) & $17(30.4 \%)$ & $21(29.6 \%)$ & $13(36.1 \%)$ & $9(37.5 \%)$ & 0.7 & 0.8 \\
\hline Preoperative laboratory tests & & & & & & \\
\hline Platelet (109/L) & $195.5(106.0)$ & $191.0(82.0)$ & $191.0(83.3)$ & $177.0(65.5)$ & 0.1 & 0.2 \\
\hline Lymphocyte (109/L) & $1.39(0.75)$ & $1.24(0.81)$ & $1.13(0.59)$ & $1.41(0.99)$ & 0.01 & 0.03 \\
\hline Monocyte (109/L) & $0.70(0.56)$ & $0.67(0.60)$ & $0.89(0.63)$ & $0.92(0.82)$ & 0.03 & 0.03 \\
\hline Neutrophil (109/L) & $7.39 \pm 3.60$ & $7.76 \pm 3.41$ & $8.97 \pm 3.33$ & $10.52 \pm 3.76$ & 0.04 & 0.001 \\
\hline Hyperlipidemia (n) & $14(25.0 \%)$ & $16(22.5 \%)$ & $3(8.3 \%)$ & $9(37.5 \%)$ & 0.6 & 0.02 \\
\hline Retinol binding protein (mg/L) & $27.6(24.1)$ & $27.9(17.1)$ & $21.2(16.4)$ & $37.3(25.2)$ & 0.7 & 0.02 \\
\hline Preoperative renal function & & & & & & \\
\hline Dissection involving renal artery $(n)$ & $4(7.1 \%)$ & $6(8.5 \%)$ & $4(11.1 \%)$ & $5(20.8 \%)$ & 0.4 & 0.3 \\
\hline Preoperative $\mathrm{SCr}(\mu \mathrm{mol} / \mathrm{L})$ & $65.5(31.0)$ & $75.0(24.4)$ & $68.5(36.2)$ & $81.1(47.9)$ & 0.2 & 0.2 \\
\hline$>106(n)$ & $6(10.7 \%)$ & $10(14.1 \%)$ & $4(11.1 \%)$ & $7(29.2 \%)$ & 0.3 & 0.2 \\
\hline Surgical details & & & & & & \\
\hline Involving the aortic arch (n) & $50(89.3 \%)$ & $66(93.0 \%)$ & $35(97.2 \%)$ & $24(100.0 \%)$ & 0.1 & 0.2 \\
\hline Involving the descending aorta (n) & $43(76.8 \%)$ & $59(83.1 \%)$ & $33(91.7 \%)$ & $23(95.8 \%)$ & 0.06 & 0.1 \\
\hline CPB duration (min) & $188.0(38.8)$ & $201.0(44.0)$ & $222.0(51.8)$ & $235.0(60.0)$ & $<0.001$ & $<0.001$ \\
\hline DHCA or MHCA (n) & $48(85.7 \%)$ & $65(91.5 \%)$ & $34(94.4 \%)$ & $23(95.8 \%)$ & 0.1 & 0.4 \\
\hline Aortic cross-clamp time (min) & $94.5(21.8)$ & $99.0(31.0)$ & $96.0(32.5)$ & $110.0(29.0)$ & 0.02 & 0.02 \\
\hline Operation time (h) & $7.0(1.5)$ & $8.0(1.5)$ & $8.5(1.9)$ & $9.0(2.5)$ & $<0.001$ & $<0.001$ \\
\hline Combined surgery & & & & & & \\
\hline CABG (n) & $1(1.8 \%)$ & $2(2.8 \%)$ & $0(0.0 \%)$ & $3(12.5 \%)$ & 0.5 & 0.04 \\
\hline Valvular surgery (n) & $8(14.3 \%)$ & $7(9.9 \%)$ & $1(2.8 \%)$ & $0(0.0 \%)$ & 0.07 & 0.1 \\
\hline Outcomes & & & & & & \\
\hline Renal replacement therapy (n) & $0(0.0 \%)$ & $0(0.0 \%)$ & $0(0.0 \%)$ & $11(45.8 \%)$ & 0.03 & $<0.001$ \\
\hline Length of ICU stay (day) & $5.0(3.8)$ & $5.0(2.0)$ & $5.0(4.0)$ & $12.0(11.5)$ & 0.02 & $<0.001$ \\
\hline$>7 d(n)$ & $17(30.4 \%)$ & $16(22.5 \%)$ & $13(36.1 \%)$ & $22(91.7 \%)$ & 0.3 & $<0.001$ \\
\hline Intubation time (h) & $19.0(20.0)$ & $24.0(32.0)$ & $40.5(75.8)$ & $147.5(81.0)$ & $<0.001$ & $<0.001$ \\
\hline$>5 d(n)$ & $4(7.1 \%)$ & $4(5.6 \%)$ & $5(13.9 \%)$ & $17(70.8 \%)$ & 0.03 & $<0.001$ \\
\hline Redo surgery (n) & $2(3.6 \%)$ & $2(2.8 \%)$ & $5(13.9 \%)$ & $6(25.0 \%)$ & 0.1 & 0.002 \\
\hline In-hospital mortality (n) & $1(1.8 \%)$ & $1(1.4 \%)$ & $0(0.0 \%)$ & $7(29.2 \%)$ & 0.2 & $<0.001$ \\
\hline
\end{tabular}

Abbreviations: $B M I$ body mass index, SCr Serum Creatinine, CPB Cardiopulmonary Bypass, DHCA Deep Hypothermia and Circulatory Arrest, MHCA Median Hypothermia and Circulatory Arrest, CABG Coronary Artery Bypass Graft

NOTE. The categorical variables in the table are presented by the number of cases (with percentage) and the continuous variables are expressed by the median (with interquartile range) or mean (with standard deviation)

* $P 1$ Value: Compare the patients without AKI and all patients with AKI. $P_{1}$ values* were the results of unpaired t-test or Mann-Whitney $\mathrm{U}$ test for continuous variables, and $\mathrm{X} 2$ test or Fisher's exact test for categorical variables

\# $P 2$ Value: Compare patients within four groups (without AKI and patients with stage 1, 2 and $3 \mathrm{AKI}) . P_{2}$ values\# were the results of one-way analysis of variance or KruskalWallis test for continuous variables, and $\mathrm{X} 2$ test or Fisher's exact test for categorical variables 
Table 4 Univariate Analysis of Risk Factors for the patients with acute aortic syndrome

\begin{tabular}{llll}
\hline Variable & Odds Ratio & $95 \%$ Confidence Interval & $P$ Value \\
\hline Age (y) & 0.98 & $0.95-1.01$ & 0.2 \\
Sex, male & 2.05 & $1.07-3.92$ & 0.03 \\
Weight & 1.04 & $1.01-1.07$ & 0.009 \\
Preoperative platelet & 1.00 & $0.99-1.00$ & 0.06 \\
Preoperative lymphocyte & 0.61 & $0.37-1.01$ & 0.05 \\
Preoperative monocyte & 2.42 & $1.10-5.36$ & 0.03 \\
Preoperative neutrophil & 1.10 & $1.01-1.21$ & 0.04 \\
NLR & 1.16 & $1.06-1.28$ & 0.002 \\
LMR & 0.77 & $0.64-0.93$ & 0.007 \\
Preoperative NYHA class III or IV & 0.39 & $0.14-1.03$ & 0.06 \\
Involving the descending aorta & 2.17 & $0.97-4.89$ & 0.06 \\
CPB duration & 1.02 & $1.01-1.03$ & $<0.001$ \\
DHCA or MHCA & 2.26 & $0.82-6.20$ & 0.1 \\
Aortic cross-Clamp time & 1.02 & $1.00-1.04$ & 0.01 \\
Operation time & 1.63 & $1.26-2.10$ & $<0.001$ \\
Combined valvular surgery & 0.39 & $0.14-1.1$ & 0.08 \\
\hline Abbristion
\end{tabular}

Abbreviations: NLR Neutrophil to Lymphocyte Ratio, LMR Lymphocyte to Monocyte Ratio, CPB Cardiopulmonary Bypass, DHCA Deep Hypothermia and Circulatory Arrest, MHCA Median Hypothermia and Circulatory Arrest

elephant trunk (FET) implantation, patients receiving surgical treatment for type A aortic dissection and so on. We believe that the deviation of AKI incidence and predictive factors from various studies on OTAS was to a large extent attributable to the heterogeneity of recruited subjects including ethnicity, disease types and surgical procedures and etc.

According to our search result, the incidence of postoperative AKI following OTAS has been rarely studied in the patients with AAS and our work was among the first to focus on this special subgroup. The definition of AAS comprises of a series of acute and catastrophic aortic lesions including acute aortic dissection, intramural hematoma, penetrating aortic ulcer, and rupture of aorta due to aortic aneurysm or trauma [14]. For most heart centers across the globe including ours, emergency aortic surgery is indicated for the patients with AAS. Even for the patients at advanced age and with co-morbidities, urgent surgery is highly recommended, and for the most circumstances is not surgically contraindicated. Of note, the patients with AAS are theoretically under a higher risk of postoperative AKI than the overall cohort and non-AAS group. First, the patients with AAS are more likely to develop into a serious situation of single- or multi-organ dysfunction including cardiac, hepatic and respiratory failure, which is tightly associated with renal dysfunction and even failure. Second, acute aortic lesion could lead to the sudden disruption of normal blood supply to renal, which inevitably results in the occurrence of AKI. Third, the systemic inflammation response syndrome (SIRS) and ischemia-reperfusion injury could further deteriorate the status of renal function and worsen the AKI. Other preoperative risk factors among AAS patients compared to non-AAS patients might include hypertension, obesity, preoperative renal injury and repeated contrast agents used in CT or MRI scan. Fourth, a majority of patients with AAS will undergo the aortic arch repair and reconstruction, which signifies the prolonged operation time, CPB time and DHCA or MHCA. Especially in China, the patients with AAS have a younger average age at disease onset than the patients in the western countries [15]. And the Chinese patients prefer to undergo a more radical surgical operation (such as Sun's procedure) for only once because of limited financial support and medical insurance. Therefore, the patients with AAS should be separately analyzed for the incidence and risk factors of postoperative AKI following OTAS. Our results revealed that compared to the non-AAS subgroup, an extra of 15\% more patients with AAS will experience the AKI following OTAS. And the prolonged $\mathrm{CPB}$ time and reduced preoperative LMR were independently associated with the occurrence of AKI in the AAS subgroup. As expected, the predictive models for AKI were different between the AAS and non-AAS subgroups. Our results were consistent with several previous studies that CPB time instead of DHCA time were statistically linked with postoperative AKI [7, 15]. It is hypothesized by Sun and this team that DHCA 
Table 5 Characteristics of the patients without acute aortic syndrome

\begin{tabular}{|c|c|c|c|c|c|c|}
\hline & No AKI & AKI Stage & & & $P 1$. & $P 2$ \\
\hline & & 1 & 2 & 3 & & \\
\hline Patient population (n) & 113 & 82 & 13 & 4 & & \\
\hline Demographic data & & & & & & \\
\hline Age (y) & $53.0(17.5)$ & $60.0(11.0)$ & $52.0(14.0)$ & $55.0(19.5)$ & 0.002 & 0.01 \\
\hline Sex, male (n) & $81(71.7 \%)$ & $64(78.0 \%)$ & $10(76.9 \%)$ & $3(75.0 \%)$ & 0.3 & 0.8 \\
\hline Height (cm) & $170.0(14.0)$ & $168.0(10.5)$ & $165.0(15.0)$ & $166.5(4.5)$ & 0.1 & 0.3 \\
\hline Weight (kg) & $67.0(16.0)$ & $69.1(15.6)$ & $66.0(15.0)$ & $62.0(17.0)$ & 0.6 & 0.2 \\
\hline $\mathrm{BMI}\left(\mathrm{kg} / \mathrm{m}^{2}\right)$ & $24.2(5.3)$ & $25.0(5.7)$ & $22.9(5.0)$ & $22.8(5.6)$ & 0.09 & 0.09 \\
\hline Medical history & & & & & & \\
\hline Diabetes $(n)$ & $2(1.8 \%)$ & $3(3.7 \%)$ & $1(7.7 \%)$ & $0(0.0 \%)$ & 0.3 & 0.6 \\
\hline Hypertension (n) & $36(31.9 \%)$ & $39(47.6 \%)$ & $4(30.8 \%)$ & $0(0.0 \%)$ & 0.08 & 0.05 \\
\hline Chronic obstructive pulmonary disease ( $\mathrm{n}$ ) & $3(2.7 \%)$ & $5(6.1 \%)$ & $1(7.7 \%)$ & $0(0.0 \%)$ & 0.2 & 0.6 \\
\hline Previous myocardial infarction (n) & $1(0.9 \%)$ & $0(0.0 \%)$ & $0(0.0 \%)$ & $0(0.0 \%)$ & 0.3 & 0.8 \\
\hline Peripheral vascular disease $(\mathrm{n})$ & $9(8.0 \%)$ & $6(7.3 \%)$ & $1(7.7 \%)$ & $0(0.0 \%)$ & 0.8 & 0.9 \\
\hline Smoking (n) & $40(35.4 \%)$ & $38(46.3 \%)$ & $6(46.2 \%)$ & $3(75.0 \%)$ & 0.08 & 0.2 \\
\hline Preoperative laboratory tests & & & & & & \\
\hline Platelet (109/L) & $197.0(57.5)$ & $183.0(58.0)$ & $203.0(54.5)$ & $137.5(109.3)$ & 0.03 & 0.09 \\
\hline Preoperative renal function & & & & & & \\
\hline $\mathrm{eGFR}(\mathrm{mL} / \mathrm{min} / 1.73 \mathrm{~m} 2)$ & $99.8(16.1)$ & $95.9(18.1)$ & $101.7(16.4)$ & $97.2(37.6)$ & 0.04 & 0.02 \\
\hline Preoperative cardiovascular status & & & & & & \\
\hline Previous cardiovascular surgery (n) & $2(1.8 \%)$ & $1(1.2 \%)$ & $0(0.0 \%)$ & $1(25.0 \%)$ & 0.9 & 0.008 \\
\hline LVEF (\%) & $58.0(8.0)$ & $60.0(4.5)$ & $52.5(17.8)$ & $59.0(11.8)$ & 0.2 & 0.08 \\
\hline Marfan syndrome (n) & $11(9.7 \%)$ & $2(2.4 \%)$ & $1(7.7 \%)$ & $0(0.0 \%)$ & 0.05 & 0.2 \\
\hline Intraoperative blood product use & & & & & & \\
\hline Erythrocytes (u) & $2.0(3.0)$ & $2.0(4.0)$ & $3.0(3.0)$ & $4.0(6.0)$ & 0.2 & 0.07 \\
\hline Fresh frozen plasma (ml) & $400.0(0.0)$ & $400.0(0.0)$ & $400.0(300.0)$ & $650.0(450.0)$ & 0.2 & 0.02 \\
\hline Platelets (u) & $1.0(1.0)$ & $1.0(1.0)$ & $1.0(1.0)$ & $2.0(0.8)$ & 0.5 & 0.004 \\
\hline Surgical details & & & & & & \\
\hline Involving the aortic arch (n) & $29(25.7 \%)$ & $31(37.8 \%)$ & $3(23.1 \%)$ & $2(50.0 \%)$ & 0.09 & 0.2 \\
\hline Involving the descending aorta $(\mathrm{n})$ & $0(0.0 \%)$ & $1(1.2 \%)$ & $0(0.0 \%)$ & $1(25.0 \%)$ & 0.1 & $<0.001$ \\
\hline CPB duration(min) & $117.5(43.0)$ & $131.0(50.5)$ & $137.0(52.5)$ & $187.5(34.8)$ & 0.005 & 0.003 \\
\hline DHCA or MHCA (n) & $10(8.8 \%)$ & $11(13.4 \%)$ & $0(0.0 \%)$ & $2(50.0 \%)$ & 0.3 & 0.03 \\
\hline Aortic cross-clamp time (min) & $81.0(27.8)$ & $83.0(31.3)$ & $94.0(32.5)$ & $122.0(42.8)$ & 0.08 & 0.04 \\
\hline Operation time (h) & $5.0(1.0)$ & $6.0(1.5)$ & $7.0(2.3)$ & $8.5(2.5)$ & $<0.001$ & $<0.001$ \\
\hline Combined surgery & & & & & & \\
\hline CABG (n) & $8(7.1 \%)$ & $11(13.4 \%)$ & $2(15.4 \%)$ & $1(25.0 \%)$ & 0.09 & 0.3 \\
\hline Outcomes & & & & & & \\
\hline Renal replacement therapy ( $n$ ) & $0(0.0 \%)$ & $0(0.0 \%)$ & $0(0.0 \%)$ & $1(25.0 \%)$ & 0.3 & $<0.001$ \\
\hline ICU length of stay (day) & $3.0(1.0)$ & $3.0(2.0)$ & $5.0(5.0)$ & $4.0(7.5)$ & $<0.001$ & $<0.001$ \\
\hline$>7 d(n)$ & $2(1.8 \%)$ & $4(4.9 \%)$ & $4(30.8 \%)$ & $1(25.0 \%)$ & 0.02 & $<0.001$ \\
\hline Intubation time (h) & $13.0(8.0)$ & $14.0(9.0)$ & $20.0(28.5)$ & $28.5(28.5)$ & 0.03 & 0.001 \\
\hline$>5 \mathrm{~d}(\mathrm{n})$ & $0(0.0 \%)$ & $1(1.2 \%)$ & $1(7.7 \%)$ & $0(0.0 \%)$ & 0.1 & 0.06 \\
\hline In-hospital mortality (n) & $1(0.9 \%)$ & $0(0.0 \%)$ & $1(7.7 \%)$ & $1(25.0 \%)$ & 0.5 & $<0.001$ \\
\hline
\end{tabular}

Abbreviations: $B M I$ body mass index, INR International Normalized Ratio, eGFR Estimated Glomerular Filtration Rate, LVEF Left Ventricular Ejection Fraction, CPB Cardiopulmonary Bypass, DHCA Deep Hypothermia and Circulatory Arrest, MHCA Median Hypothermia and Circulatory Arrest, CABG Coronary Artery Bypass Graft

NOTE. The categorical variables in the table are presented by the number of cases (with percentage) and the continuous variables are expressed by the median (with interquartile range) or mean (with standard deviation)

* $P 1$ Value: Compare the patients without AKI and all patients with AKI. $P_{1}$ values* were the results of unpaired t-test or Mann-Whitney $U$ test for continuous variables, and $\mathrm{X} 2$ test or Fisher's exact test for categorical variables

\#P2 Value: Compare patients within four groups (without AKI and patients with stage 1,2 and $3 \mathrm{AKI}$ ). $P_{2}$ values\# were the results of one-way analysis of variance or KruskalWallis test for continuous variables, and $\mathrm{X} 2$ test or Fisher's exact test for categorical variables 
Table 6 Univariate Analysis of Risk Factors for the patients without acute aortic syndrome

\begin{tabular}{llll}
\hline Variable & Odds Ratio & $95 \%$ Confidence Interval & $P$ Value \\
\hline Age (y) & 1.05 & $1.02-1.08$ & $<0.001$ \\
Sex, male & 1.38 & $0.74-2.59$ & 0.3 \\
Hypertension & 1.64 & $0.94-2.88$ & 0.08 \\
Smoking & 1.65 & $0.95-2.86$ & 0.08 \\
Preoperative platelet & 0.99 & $0.99-1.00$ & 0.02 \\
PLR & 0.99 & $0.99-1.00$ & 0.05 \\
Preoperative eGFR & 0.98 & $0.97-1.00$ & 0.06 \\
Marfan syndrome & 0.29 & $0.08-1.07$ & 0.06 \\
Involving the aortic arch & 1.66 & $0.92-2.98$ & 0.09 \\
CPB duration & 1.01 & $1.00-1.02$ & 0.01 \\
Operation time & 1.56 & $1.22-1.99$ & $<0.001$
\end{tabular}

Abbreviations: PLR Platelet to Lymphocyte Ratio, eGFR Estimated Glomerular Filtration Rate, $C P B$ Cardiopulmonary Bypass

could significantly extend the $\mathrm{CPB}$ time, which is likely to be counterbalanced by the protective effects of DHCA on renal function. Additionally, the preoperative LMR might also function as a novel bio-marker for predicting postoperative AKI following OTAS, and such report has not been published so far and it awaits further investigation.

Our team also analyzed the data from the non-AAS subgroup in whom elective OTAS were performed. Englberger and his colleagues carried out a retrospective study with a larger sample size on elective OTAS [2]. However, our

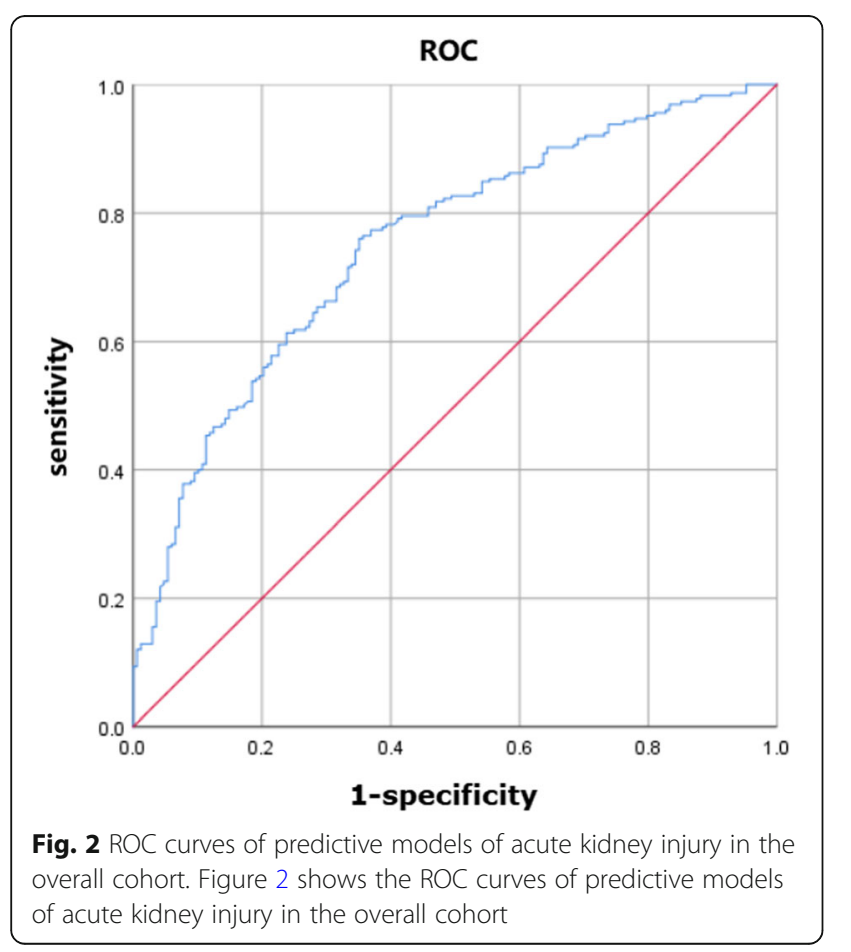

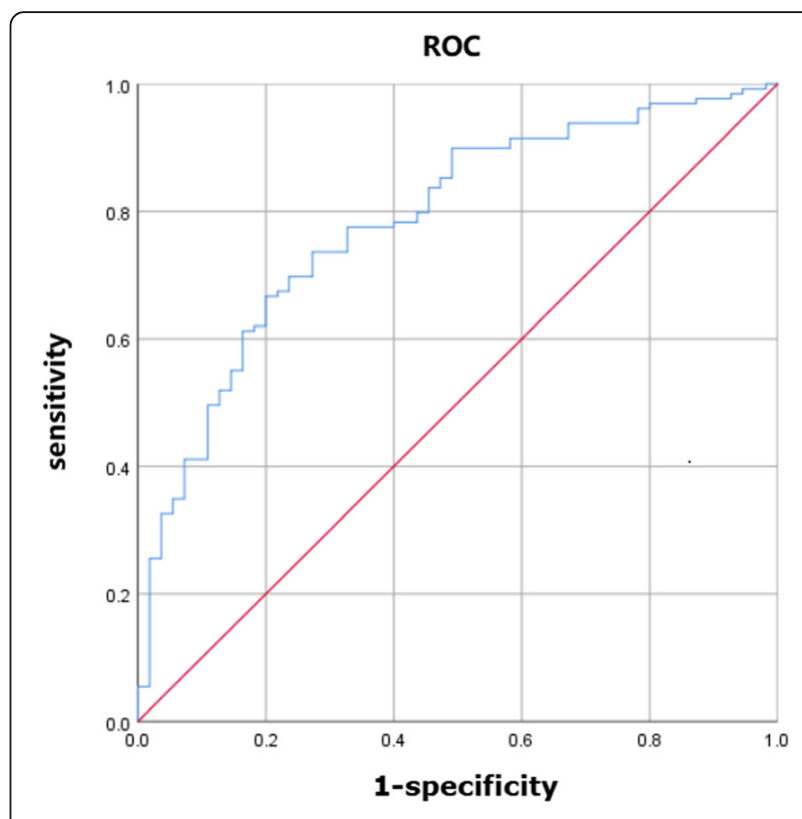

Fig. 3 ROC curves of predictive models of acute kidney injury in the AAS subgroup. Figure 3 shows the ROC curves of predictive models of acute kidney injury in the AAS subgroup

work was the first to investigate the AKI following elective OTAS in Chinese ethnicity though the sample size was relatively small. Besides, the results of diagnostic test suggested that our predictive model for AKI after OTAS has a good predictive ability, with its sensitivity and specificity to be further improved in the future.

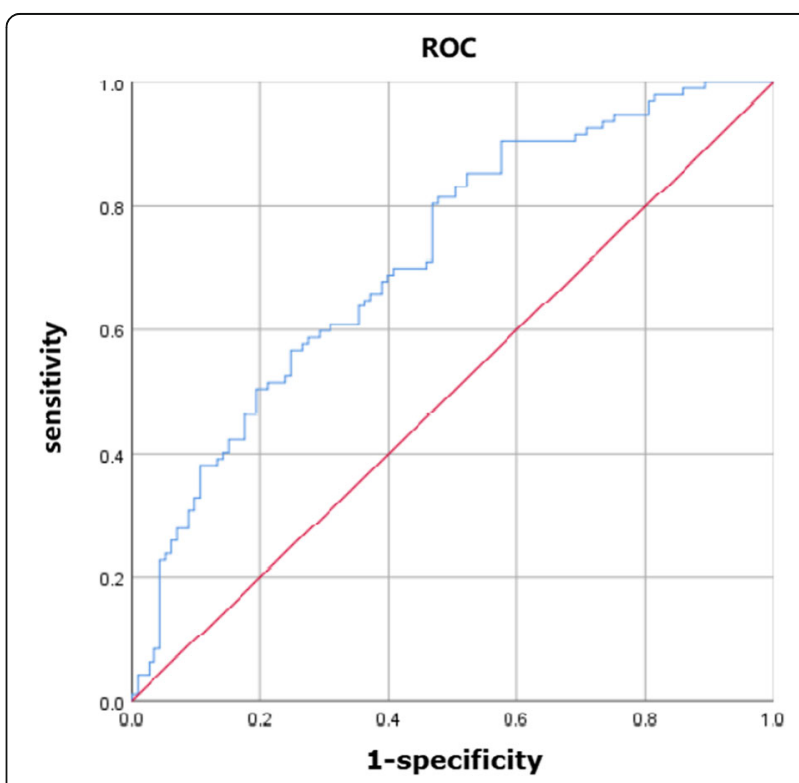

Fig. 4 ROC curves of predictive models of acute kidney injury in the non-AAS subgroup. Figure 4 shows the ROC curves of predictive models of acute kidney injury in the non-AAS subgroup 


\section{Conclusions}

The patients with AAS have a higher risk of developing postoperative AKI after OTAS compared with those without AAS. The predictive factors for postoperative AKI following OTAS vary between AAS and non-AAS subgroups. Operation time, $\mathrm{CPB}$ time and preoperative platelet are modifiable predictors of AKI.

\section{Supplementary information}

Supplementary information accompanies this paper at https://doi.org/10 1186/s13019-020-01257-1.

\section{Additional file 1}

Additional file 2. Supplementary Table 1 Surgical options of the overall cohort

Additional file 3. Supplementary Table 2 Surgical options of AAS patient

Additional file 4. Supplementary Table 3 Surgical options of non-AAS patients

\section{Abbreviations}

AKl: Acute kidney injury; OTAS: Open thoracic aortic surgery; AAS: Acute aortic syndrome; CPB: Cardiopulmonary bypass; RRT: Renal replacement therapy; GCP: Good Clinical Practice; KDIGO: Kidney Disease: Improving Global Outcomes guidelines; Scr: Serum creatinine; SD: Standard deviation; ROC: Receiver operating curve; NLR: Neutrophil to lymphocyte ratio; LMR: Lymphocyte to monocyte ratio; PLR: Platelet to lymphocyte ratio; TAR: Total aorta replacement; FET: Frozen elephant trunk; SIRS: Systemic inflammation response syndrome

\section{Acknowledgements}

Not applicable.

\section{Authors' contributions}

$Y C$ and $X M$ designed the research. $X M, J L, Y Y, D Z, S C$ and $H M$ analyzed the data. $X M, J L$ and $Y Y$ wrote the paper. $Z W, H Z$ and $C Z$ were responsible for data collection. All authors read and approved the final manuscript.

\section{Funding}

The current work was supported by grants from The National Nature Science Foundation of China (grant no. 81600222 to YC and 81800255 to MX), Young experts of Taishan Scholar Program of Shandong Province to YC (tsqn201812142) and Natural Science Foundation of Shandong Province (ZR2018BH002 to MX). The funders had no role in study design, data collection and analysis, decision to publish, or preparation of the manuscript.

\section{Availability of data and materials}

The datasets used and/or analyzed during the current study are available from the corresponding author on reasonable request.

\section{Ethics approval and consent to participate}

This single-center retrospective cohort study was approved by the Ethics Committee of Shandong Provincial Hospital and the written informed consent was waived due to the retrospective design.

\section{Consent for publication}

Consents for publication were obtained from all individuals involved in our study.

\section{Competing interests}

The authors declare that they have no competing interests.

\section{Author details}

'Department of Cardiovascular Surgery, Shandong Provincial Hospital affiliated to Shandong First Medical University, No.324 Jingwu Road, Jinan 250021, Shandong, China. ${ }^{2}$ Department of Cardiovascular Surgery, Shandong
Provincial Hospital affiliated to Shandong University, No.324 Jingwu Road, Jinan 250021, Shandong, China. ${ }^{3}$ College of Basic Medicine, Capital Medical University, Beijing, China. ${ }^{4}$ Department of Radiology, Qilu Hospital of Shandong University, No.107 West Wenhua Road, Jinan 250012, Shandong, China. ${ }^{5}$ Qingdao University Medical College, Qingdao University, 308 Ningxia Road, Qingdao 266071, Shandong, China. 'Department of Cardiology, Shandong Provincial Hospital affiliated to Shandong First Medical University, No.324 Jingwu Road, Jinan 250021, Shandong, China. ${ }^{7}$ Center for Precision Medicine and Division of Cardiovascular Medicine, University of Missouri School of Medicine, Columbia, MO 65212, USA

Received: 22 April 2020 Accepted: 28 July 2020

Published online: 07 August 2020

\section{References}

1. Ko T, Higashitani M, Sato A, Uemura Y, Norimatsu T, Mahara K, et al. Impact of acute kidney injury on early to long-term outcomes in patients who underwent surgery for type a acute aortic dissection. Am J Cardiol. 2015; 116(3):463-8.

2. Englberger L, Suri RM, Greason $K L$, Burkhart HM, Sundt TM 3rd, Daly RC, et al. Deep hypothermic circulatory arrest is not a risk factor for acute kidney injury in thoracic aortic surgery. J Thorac Cardiovasc Surg. 2011; 141(2):552-8.

3. Zhao H, Pan X, Gong Z, Zheng J, Liu Y, Zhu J, et al. Risk factors for acute kidney injury in overweight patients with acute type a aortic dissection: a retrospective study. J Thorac Dis. 2015;7(8):1385-90.

4. Kim WH, Lee JH, Kim E, Kim G, Kim HJ, Lim HW. Can we really predict postoperative acute kidney injury after aortic surgery? Diagnostic accuracy of risk scores using gray zone approach. Thorac Cardiovasc Surg. 2016;64(4):281-9.

5. Roh GU, Lee JW, Nam SB, Lee J, Choi JR, Shim YH. Incidence and risk factors of acute kidney injury after thoracic aortic surgery for acute dissection. Ann Thorac Surg. 2012;94(3):766-71

6. Mori Y, Sato N, Kobayashi Y, Ochiai R. Acute kidney injury during aortic arch surgery under deep hypothermic circulatory arrest. J Anesth. 2011;25(6):799-804.

7. Nota H, Asai T, Suzuki T, Kinoshita T, Ikegami H, Takashima N. Risk factors for acute kidney injury in aortic arch surgery with selective cerebral perfusion and mild hypothermic lower body circulatory arrest. Interact Cardiovasc Thorac Surg. 2014;19(6):955-61.

8. Hobson CE, Yavas S, Segal MS, Schold JD, Tribble CG, Layon AJ, et al. Acute kidney injury is associated with increased long-term mortality after cardiothoracic surgery. Circulation. 2009;119(18):2444-53.

9. D'Onofrio A, Cruz D, Bolgan I, Auriemma S, Cresce GD, Fabbri A, et al. RIFLE criteria for cardiac surgery-associated acute kidney injury: risk factors and outcomes. Congest Heart Fail. 2010;16(Suppl 1):S32-6.

10. Arnaoutakis GJ, Bihorac A, Martin TD, Hess PJ Jr, Klodell CT, Ejaz AA, et al. RIFLE criteria for acute kidney injury in aortic arch surgery. J Thorac Cardiovasc Surg. 2007;134(6):1554-60 discussion 60-1.

11. Kowalik MM, Lango R, Klajbor K, Musial-Swiatkiewicz V, Kolaczkowska M, Pawlaczyk R, et al. Incidence- and mortality-related risk factors of acute kidney injury requiring hemofiltration treatment in patients undergoing cardiac surgery: a single-center 6-year experience. J Cardiothorac Vasc Anesth. 2011;25(4):619-24.

12. Bove T, Calabro MG, Landoni G, Aletti G, Marino G, Crescenzi G, et al. The incidence and risk of acute renal failure after cardiac surgery. J Cardiothorac Vasc Anesth. 2004:18(4):442-5.

13. Kellum JA, Lameire N, Group KAGW. Diagnosis, evaluation, and management of acute kidney injury: a KDIGO summary (part 1). Crit Care. 2013;17(1):204

14. Erbel R, Aboyans V, Boileau C, Bossone E, Di Bartolomeo R, Eggebrecht H, et al. 2014 ESC guidelines on the diagnosis and treatment of aortic diseases. Kardiol Pol. 2014;72(12):1169-252.

15. Zhou H, Wang G, Yang L, Shi S, Li J, Wang M, et al. Acute kidney injury after Total arch replacement combined with frozen elephant trunk implantation: incidence, risk factors, and outcome. J Cardiothorac Vasc Anesth. 2018;32(5): 2210-7.

\section{Publisher's Note}

Springer Nature remains neutral with regard to jurisdictional claims in published maps and institutional affiliations. 